\title{
Object Detection in a Cluttered Scene Using SURF for Computer Assisted Histopathology
}

\author{
Aqeel Abbas ${ }^{1, *}$, Talat $\mathrm{Zehra}^{2}$ and $\mathrm{Fu} \mathrm{Li}^{3}$ \\ ${ }^{1} \mathrm{PhD}$ scholar, Beihang University, Beijing, China \\ ${ }^{2}$ Histopathologist PN Shifa, Karachi, Pakistan \\ ${ }^{3}$ Supervisor, Beihang University, Beijing, China \\ *Corresponding author
}

\begin{abstract}
Computer vision imitates human vision ability to perceive real world. Computer vision has proven its part from autonomous navigation on martin surface to histopathology images recognition. In practice, histopathologists examine microscopic imagery of diseased tissue and diagnose on the bases of malignancy, likewise a computer assisted diagnosis (CAD) system is devised for histopathology images recognition on the basis of visual features. This paper describes the design methodology, workflow and test results of locating an object in a cluttered scene. In this paper speeded up robust features are used for feature extraction and matching. After object detection, Random sample consensus is used for removing outliers to refine results. This CAD system provides a heuristic approach to assist histopathologists in diagnostics.
\end{abstract}

Keywords-computer vision; surf; ransac; computer assisted diagnostics; histopathology

\section{INTRODUCTION}

Histopathology is the study of the microscopic anatomy of cells and tissues for diagnosis. Histopathological analysis is performed by examining thin slices of tissues under microscope. In present research, the study of histopathology images is commonly regarded as the gold standard for the clinical diagnosis. In practice, histopathologists visually examine the irregularities of cell shapes and tissue distributions and determine cancerous regions and malignancy degree [4].

Manual analysis of histology tissues in current days still remains to be the primary way to identify malignancy, which depends heavily on the expertise and experience of histopathologists. Such manual intervention has the disadvantages of;

(a) Being very time consuming for such high throughput and high content screening.

(b) Being difficult to grade in a reproducible manner due to the intra- and inter-observation variations in the grading process.

Because of the large and increasingly impractical demands on expert's time to interpret the images, computer assisted diagnosis (CAD) systems for histopathology image analysis are becoming increasingly crucial in diagnosis. Compared to manual analysis, computer-based systems provide instant and consistent diagnosis [4].

\section{RELATED WORK}

Computer vision has been used in a wide verity of applications over the years, like BMW's parking assistant and NASA's Mars rover. Computer assisted diagnosis has become one of the major research subject in medical imaging and diagnosis [2].

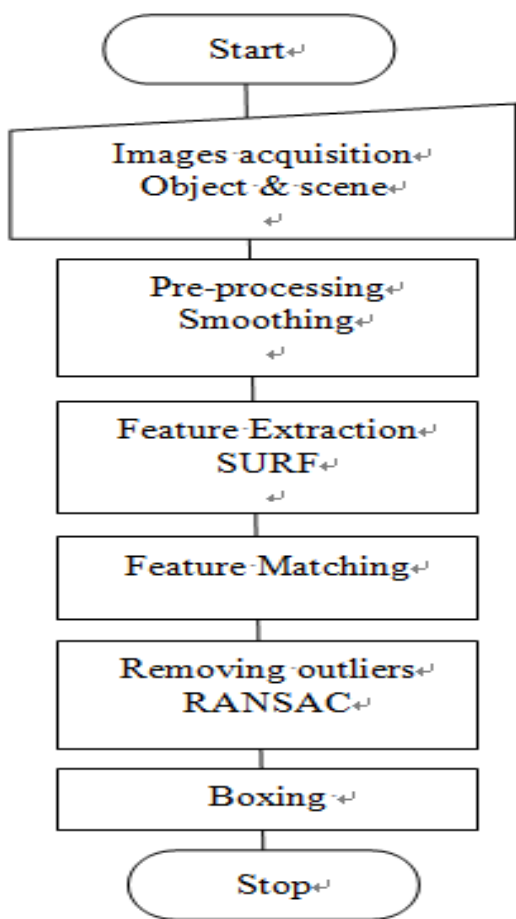

FIGURE I. WORK FLOW FOR OBJECT DETECTION IN CLUTTERED SCENE

In the paper, Validation of Digital Pathology in a Healthcare Environment, published in 2011, Amanda Lowe et al. provided a high level overview to be considered while aligning different resources available to pathologists for diagnostics [3].

In the paper, Bag-of-Visual-Ngrams for Histopathology Image Classification, Pastor et al. proposed an extension to Bag-of-visual-words for image classifier of histopathological images by representing the images as histogram of visual ngrams. $\mathrm{N}$-grams are sequences of $\mathrm{n}$ - words [5]. 

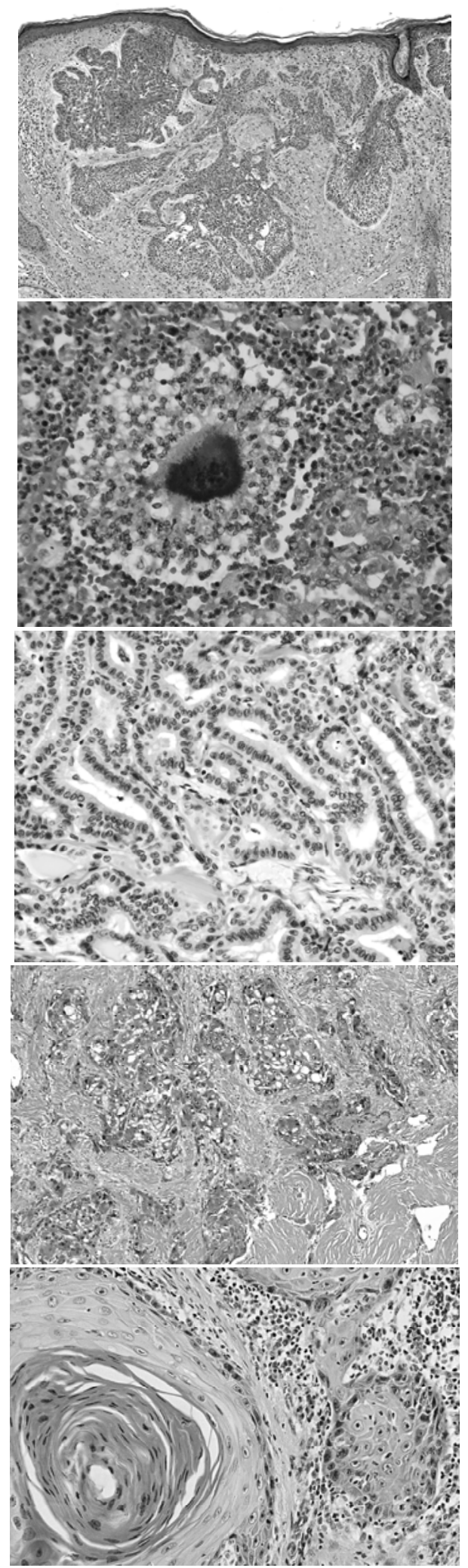

FIGURE II. HISTOPATHOLOGY IMAGES

Basal cell carcinoma, Actinomycosis, Thyroid papillary carcinoma Fibrolamellar hepatocellular carcinoma, Lung squamous carcinoma
Reference [9] provides detailed description of literature in CAD, emphasizing the role of tissue texture, also provides review on image processing tools for feature extraction, classification and validation for cancer detection and grading.

\section{COMPUTER ASISTED DIAGNOSIS}

Computer vision extends image processing for using images and videos to understand the real world scene by detecting, classifying and identifying objects and events.

After the tissue preparation and image production through digital microscope, the resulting digital histology images is examine by CAD systems as it is done by histopathologist manually. As indicated in Section I, manual histology analysis is generally very time consuming depending on the experience levels of the histopathologist. In addition, the inconsistent and variable responses from different histopathologists, including both intra- and inter-observer variations, are not uncommon in practice. To ameliorate these problems, a new CAD system is proposed to assist histopathologist with the goal of instant and consistent diagnosis. Proposed CAD system for histology image analysis is based on computer vision, which includes preprocessing, image segmentation, feature extraction, feature dimension reduction, feature matching, outlier rejection, and post-processing.

\section{ViSUAL FEATURES}

Visual features are interest parts of an image that are repeatable and distinct, which leads to an unambiguous match in other images. In this paper, Surf features are used. SURF is a scale and rotation invariant feature descriptor.

\section{FEATURE EXTRACTION}

After image segmentation, image features are extracted from the regions of interest to detect malignancy. This is implemented by applying CAD systems to identify particular disease signatures with their image features. CAD systems for histopathology image analysis in general exploit a large number of features to derive clinically significant information.

Features extracted from large size histology image result vast quantity of data, which can be prohibitive for feasible analysis, even with current high performance computing machines. Therefore, feature dimensionality reduction scheme is applied to select the most discriminative features.

\section{EXPERIMENTAL SETTINGS}

Initially we demonstrate our methodology on domestic items for straightforward understanding. A common person can perceive the following scene and classify, identify and find out the desired object in the cluttered scene. 
VII. WORK FLOW

A. Images Acqusition

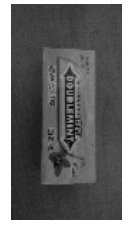

Object \& Scene

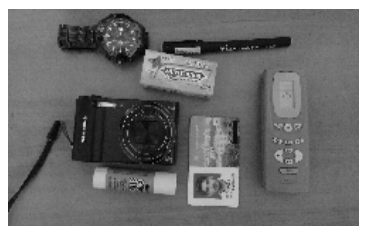

FIGURE III. DESIRED OBJECT AND CLUTTERED SCENE

\section{B. Feature Extraction}

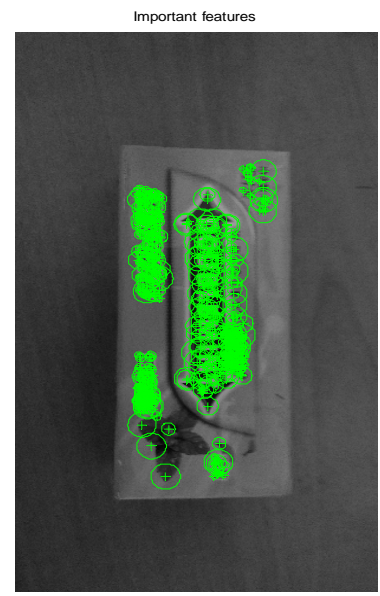

FIGURE IV. IMPORTANT SURF FEATURES

\section{Feature Matching}

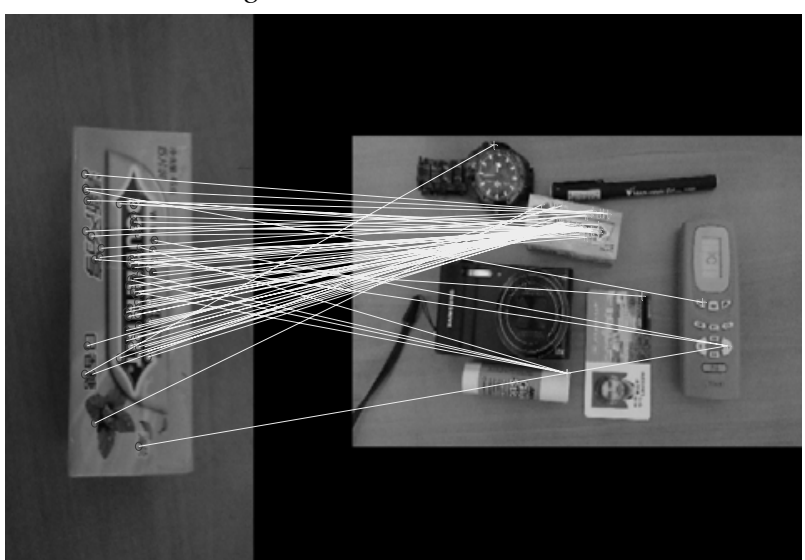

FIGURE V. MATCHED SURF FEATURES WITH OUTLIERS

\section{Outlier Removal}

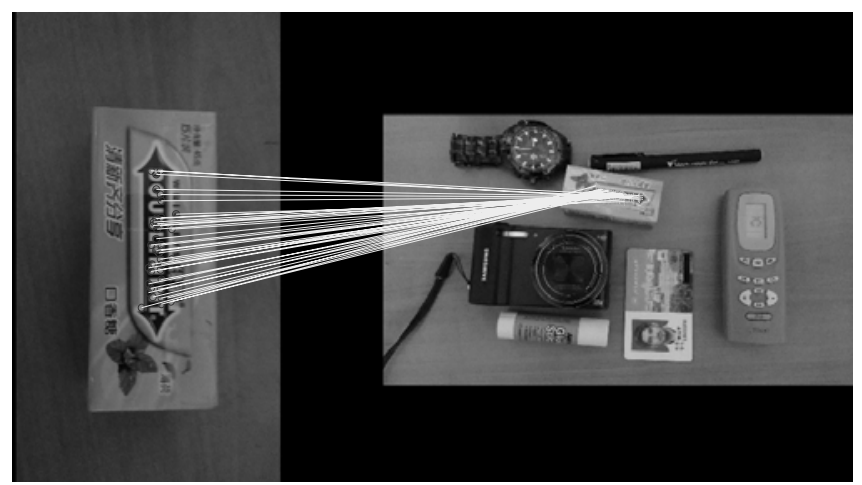

FIGURE VI. MATCHED FEATURES AFTER APPLYING RANSAC

\section{RESULTS}

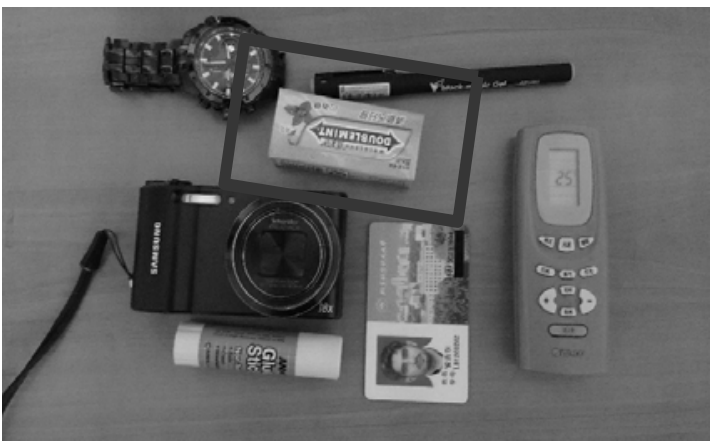

FIGURE VII. BOXING

\section{A. Histopathology Results}

Histopathologists visually analyz histopathology images to identify, classify and diagnose using their experience. Likewise our proposed $\mathrm{CAD}$ system uses visual features to spot malignant region in the slide.

Figure. 7 is a sample of Glomus tumor, our CAD system matches visual features of the sample to find out the corresponding malignant region in the histopathology slide.

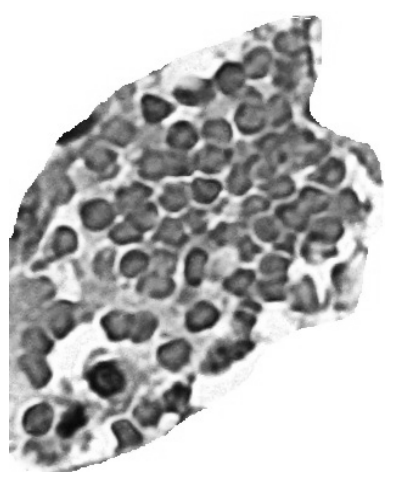

FIGURE VIII. GLOMUS TUMOR SAMPLE 


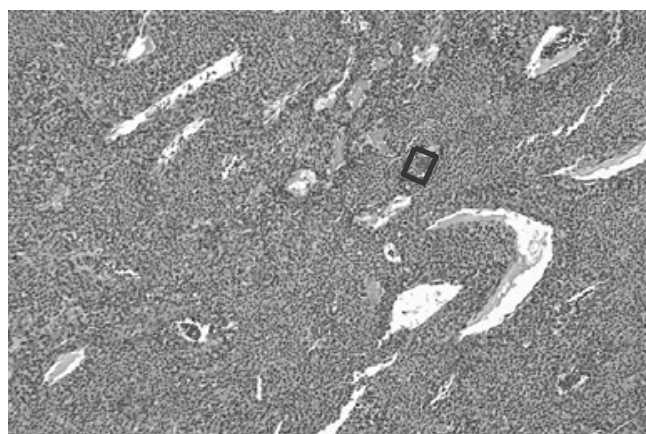

FIGURE IX. GLOMUS TUMOR HIGHLIGHTED ON THE SLIDE

\section{B. Another Histopathology Result}

In another case, CAD system is examined for the identification of crypt abscess on a histopathology slide.

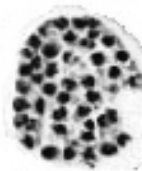

FIGURE X. CRYPT ABSCESS

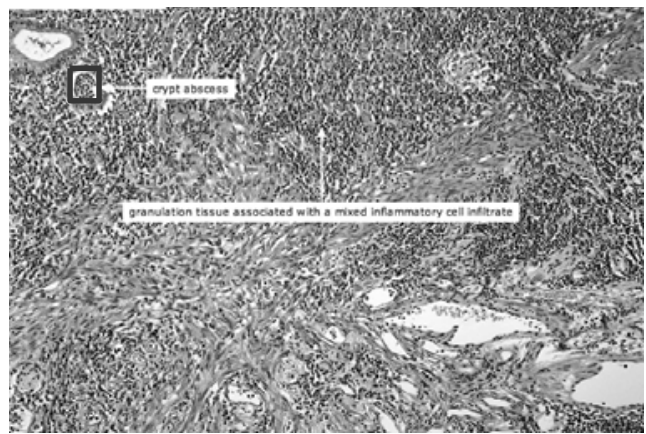

FIGURE XI. CRYPT ABSCESS HIGHLIGHTED ON SLIDE

\section{CONCLUSION}

Experimental results are demonstrated for the diagnosis of Glomus tumor and Crypt abscess via Computer assisted diagnostics using computer vision techniques. Computer assisted diagnosis is a potential field of research to assist histopathologists in instant and consistent diagnosis.

\section{FUTURE WORK}

More sophisticated trained descriptors using support vector machine can give more robust and reliable results.

\section{ACKNOWLEDGMENT}

We are grateful to Dr. Talat Zehra consultant histopathologist for her kind support.

\section{REFERENCES}

[1] Herbert Bay and Tinne Tuytelaars, "SURF: Speeded Up Robust Features", J. ECCV, 2006.

[2] Kunio Doi, "Computer-Aided Diagnosis in Medical Imaging: Historical Review, Current Status and Future Potential", Comput Med Imaging Graph. 2007 ; 31(4-5): 198-211

[3] Amanda Lowe, Elizabeth Chlipala, Jesus Elin, "Validation of Digital Pathology In a Healthcare Environment”, 2011

[4] Lei He, L. Rodney Long, Sameer Antani, "Computer Assisted Diagnosis in Histopathology", National Institutes of Health, USA

[5] A. Pastor Lopez-Monroy, Manuel Montes-y-Gomez, Hugo Jair Escalante, Angel Cruz-Roa, Fabio A. Gonzalez, "Bag-of-Visual-Ngrams for Histopathology Image Classification",

[6] Hang Chang, Yin Zhou, Paul Spellman, Bahram Parvin, "Stacked Predictive Sparse Coding for Classification of Distinct Regions in Tumor Histopathology", ICCV, 2013, pp. 169-176

[7] Ivor Kramer, "Computer-aided analyses in diagnostic histopathology", Postgraduate Medical Journal 51, pp 690-694

[8] Thomas Josef Fuchs, "Computational Pathology, A Machine Learning Approach", PhD desertation, 2010

[9] Mosquera-Lopez, Agaian, Velez-Hoyos, Thompson, "Computer-aided Prostate Cancer Diagnosis from Digitized Histopathology: A Review on Texture-based Systems", Biomedical Engineering, IEEE, 2014

[10] Arunachalam, Sasikala, Jagannathan, "Automatic detection of malignant cells in soft tissues from microscopic images", Advances in Engineering, Science and Management (ICAESM), 2012 